\title{
Sistema automático para a inspeção visual de transportadores de correia ${ }^{\star}$
}

\author{
André A. Santos* Filipe Augusto S. Rocha ${ }^{* * * * * *}$ \\ Héctor Azpúrua ${ }^{* * \dagger}$ Agnaldo J. da Rocha Reis ${ }^{* * *}$ \\ Frederico G. Guimarães ${ }^{\ddagger}$ \\ * Mestrado Profissional em Instrumentação, Controle e Automação, \\ Universidade Federal de Ouro Preto, MG, Brasil (e-mail: \\ andre.almdsantos@gmail.com) \\ ** Instituto Tecnológico Vale (ITV), MG, Brasil (email: filipe.rocha, \\ hector.azpurua@itv.org). \\ *** Universidade Federal de Ouro Preto (UFOP), MG, Brasil (email: \\ reis@ufop.edu.br). \\ **** COPPE - Universidade Federal do Rio de Janeiro, RJ, Brasil. \\ $\dagger$ VeRLab - Universidade Federal de Minas Gerais, MG, Brasil. \\ $¥$ Machine Intelligence and Data Science Laboratory (MINDS), \\ Universidade Federal de Minas Gerais, MG, Brasil (e-mail: \\ fredericoguimaraes@ufmg.br).
}

\begin{abstract}
In the mining sector, conveyor belts are the most widespread means of transportation for large quantities of materials. Mining sites usually contain thousands of bearings, which make the inspection task complex. One aims with this work to perform a research in the field of artificial intelligence in order to automate and to facilitate the inspection in conveyor belts. The proposed methodology involves training a pattern detector through Convolutional Neural Networks (CNN) from RGB images that will be collected by an autonomous robot. As an initial application, one seeks to develop a classifier capable of identifying the clutter of dirt in the structures, which is one of the tasks of the maintenance teams. To analyze the problem, we chose the use of the transfer learning technique, using networks consolidated in the classification of images and re-trained with the images collected. Accuracy in test data ranged from $81.81 \%$ to $95.45 \%$. A full description of the methodology employed and the results obtained is presented in the article.

Resumo: No setor da mineração os transportadores de correia constituem o meio mais difundido de transporte para grandes quantidades de materiais. Os locais de mineração geralmente contêm milhares de rolamentos, que tornam complexa a tarefa de inspeção. Se objetiva com este trabalho realizar uma pesquisa no campo da inteligência artificial de forma a automatizar e facilitar a inspeção em transportadores de correia. A metodologia proposta envolve treinar um classificador de padrões por meio de Redes Neurais Convolucionais (RNC) a partir de imagens RGB que serão coletadas por um robô autônomo. Como aplicação inicial, busca-se desenvolver um classificador capaz de identificar a aglomeração de sujeira nas estruturas, que é uma entre as tarefas das equipes de manutenção. Para análise do problema, optou-se pelo uso da técnica de transferência de conhecimento, usando redes consolidadas na classificação de imagens e re-treinadas com as imagens coletadas. A acurácia nos dados de teste variou entre $81.81 \%$ e $95.45 \%$. Uma descrição completa da metodologia empregada e dos resultados obtidos é apresentada no artigo.
\end{abstract}

Keywords: Conveyor Belt, Computer Vision, Artificial Neural Networks, Machine Learning. Palavras-chaves: Correia Transportadora, Visão Computacional, Redes Neurais Artificiais, Aprendizado de Máquina. 


\section{INTRODUÇÃO}

O Brasil possui uma das maiores reservas minerais do mundo, sendo um dos principais produtores e exportadores de minério de ferro. Nesse contexto, a Vale é a maior produtora mundial de minério de ferro e pelotas, matériasprimas essenciais para a fabricação do aço (Vale, 2019b). Desse modo, tecnologias que permitam melhorar as atividades relacionadas à extração, transporte e/ou venda de minérios é de grande interesse no cenário nacional, de acordo com Toffolo (2009).

No setor mínero-metalúrgico, observa-se que o Transportador de Correia (TC) é um dos meio mais difundido para transporte de grandes quantidades de materiais a granel, reduzindo dessa forma a quantidade de caminhões e o custo desse tipo de operação (Malagoni and dos Santos, 2014). A título de exemplo, a Vale possui $\approx 1200 \mathrm{~km}$ de TC. Somente em Carajás - a maior mina de minério de ferro do mundo - são $85 \mathrm{~km}$ desta estrutura (Vale, 2019a). Guastala (2013) cita o TC como um dos ativos mais importantes da empresa. Para Malagoni and dos Santos (2014), o TC detém uma posição dominante no transporte de materiais devido às suas inerentes vantagens, como economia, segurança de operação e confiabilidade.

No entanto, os TC podem apresentar problemas causando paradas não programadas, o que gera altos custo de manutenção e perda de produção. As falhas em roletes são bastante comuns. Mesmo assim, as empresas ainda possuem pouca informação sobre a vida útil e características mecânicas desses equipamentos (Loures, 2010). As plantas geralmente contêm milhares de rolamentos, sendo infactível inspecionar rentavelmente todos os rolos através do monitoramento de vibração - método mais utilizado pelas empresas segundo Loures (2010) e Matthee (2009).

Considerando que os TC transportam materiais abrasivos aglutinantes, entre outros, a vida útil dos TC é reduzida, também, em função do acúmulo desses materiais (Eletrobrás et al., 2009). Neste sentido, é interessante que soluções inteligentes sejam desenvolvidas para tratar este problema, prevenindo possíveis falhas técnicas e/ou paradas desnecessárias da planta.

O objetivo deste trabalho é desenvolver um sistema de Inteligência Artificial (IA) por RNC para detectar o acúmulo de materiais aglutinantes em estruturas de TC. O estudo está em fase preliminar, de forma que a análise por RNC evidencie a viabilidade do desenvolvimento e continuidade do projeto. Desta forma, será possível propor novas ações de manutenção baseadas na resposta deste sistema inteligente.

Será apresentado a seguir, na seção 2, uma introdução à teoria sobre CNN. A seção 3 mostra os trabalhos relacionados na área de visão computacional. Na seção 4 é apresentado a metodologia utilizada para a coleta inicial de dados e o processo de treinamento e classificação da RNC. Na seção 5 será apresentado os primeiros resultados encontrados com o conjunto da dados inicial da pesquisa. Por fim, na seção 6, serão apresentadas as principais con-

\footnotetext{
^ O presente trabalho foi realizado com apoio da Coordenação de Aperfeiçoamento de Pessoal de Nível Superior - Brasil (CAPES), CNPq e Instituto Tecnológico Vale (ITV).
}

clusões e as perspectivas para os trabalhos futuros que serão desenvolvidos.

\section{REDES NEURAIS CONVOLUCIONAIS}

O cérebro é um computador altamente complexo, não linear e paralelo que trabalha de uma forma diferente dos computadores digitais. Essa complexidade e alta capacidade de reconhecimento de padrões tem motivado os trabalhos envolvendo redes neurais ao longo dos anos. Em termos gerais, uma rede neural artificial é uma máquina projetada para modelar a forma como o cérebro realiza uma tarefa ou função em particular (Haykin et al., 2009).

$\mathrm{Na}$ identificação de padrões por imagens, as redes neurais comumente utilizam um número muito grande de neurônios, dificultando o seu uso prático. Para Aghdam and Heravi (2017), o uso de RNC é a solução para reduzir esse alto número de neurônios e parâmetros necessários para classificação de imagens.

De acordo com Haykin et al. (2009), uma RNC é uma rede multicamadas projetada especificamente para reconhecer formas bidimensionais com um alto grau de invariância à translação, escalonamento, inclinação e outras formas de distorção. Ela apresenta as seguintes restrições: Feature extraction, Feature mapping e Subsampling.

Uma imagem é composta por uma matriz de pixels. Cada pixel possui uma alta correlação com seus vizinhos e uma baixa correlação com os pixels distantes. Uma RNC aproveita esta característica para extrair informações de uma imagem por meio de filtros que são aplicados em regiões da imagem. Cada neurônio está ligado ao resultado da aplicação de um filtro em um conjunto de pixels da imagem, conforme Figura 1. A aplicação dos filtros reduz drasticamente a quantidade de neurônios necessários para o processamento da RNC, pois os pesos sinápticos de cada neurônio são compartilhados por um mesmo filtro (Aghdam and Heravi, 2017).

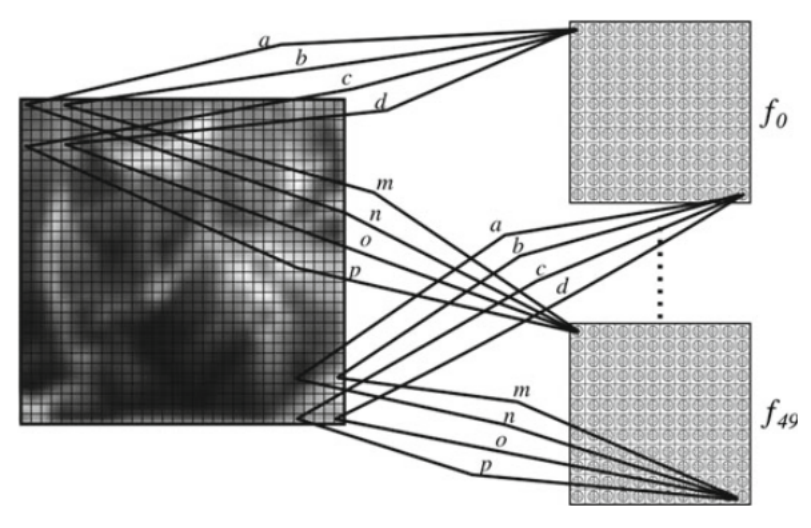

Figura 1. Extração de Características de Imagem. (Aghdam and Heravi, 2017)

A Figura 2 apresenta um esquema de RNC para reconhecimento de caracteres escritos a mão com uma camada de entrada, quatro camadas intermediárias responsáveis por extrair as características principais das imagens e uma camada de saída. 


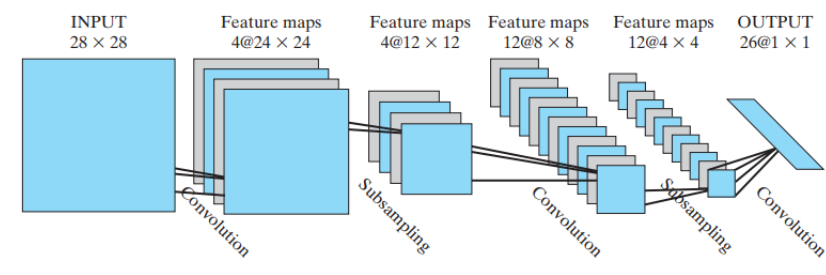

Figura 2. Rede Neural Convolucional (Haykin et al., 2009).

\section{TRABALHOS RELACIONADOS}

Algumas abordagens com o uso de IA e processamento de imagens são descritas em (Máthé and Buşoniu, 2015), onde foi realizada uma pesquisa sobre vários métodos e plataformas baratas para a inspeção visual de estruturas.

No trabalho de Luque-Vega et al. (2014) foi realizado uma pesquisa sobre inspeção de linhas aéreas de transmissão de energia via visão computacional com um sistema de voo não tripulado. A plataforma desenvolvida processa imagens de infravermelho para realizar a análise qualitativa dos componentes nas linhas de transmissão. O qualitativo de inspeção está relacionado com a temperatura relativa dos equipamentos da linha de energia e é suficiente para determinar algumas falhas comuns no sistema.

Zou and Huang (2015) e Janssens et al. (2015) apresentam métodos de identificação de problemas em equipamentos e máquinas elétricas por visão computacional. As características de temperatura e área são extraídas das imagens e usadas como entrada de um sistema baseado em IA para classificação das regiões com defeito.

A visão computacional é utilizada também para a identificação visual de problemas específicos em superfícies defeituosas. Masci et al. (2012) apresentam uma abordagem de RNC Max-Pooling com treinamento supervisionado para classificação de defeitos em superfície de aço. Com uma base de dados de uma linha de produção real foi obtido uma taxa de erro na classificação de $7 \%$, trabalhando diretamente nas intensidades dos pixels de defeito do aço detectados e segmentados.

Já no trabalho de Chen et al. (2018) é proposto o uso de uma RNC multidimensional para detectar problemas diversificados em superfícies de placas fotovoltaicas. Seis tipos de problemas diferentes foram estudados com imagens das superfícies das placas e imagens no espaço de cores RGB.

A classificação da textura do solo, baseado em dados hiperespectrais com redes RNC é objeto de estudo de Riese and Keller (2019). Foram comparados seis classificadores diferentes e analisados os parâmetros de validação das redes. Entre elas, as RNC apresentaram resultados semelhantes de classificação.

Em mineração, Qiao et al. (2013) apresentam uma proposta para testar eficientemente a falha do rasgo longitudinal de uma correia. Eles propõem um método de detecção por imagem infravermelha. No trabalho foi pesquisado a relação entre o rasgo longitudinal e o número de pixels da área rasgada detectada na imagem. Desse modo, eles estabeleceram uma tabela de limiar para análise de imagens infravermelhas anti-coloridas com resolução de $256 \times 256$ pixels.

\section{METODOLOGIA}

Esta seção apresenta a metodologia para a coleta de dados e desenvolvimento do processo de treinamento. Todo o processo de desenvolvimento da RNC foi realizado em Python utilizando a biblioteca Pytorch, (Paszke et al., 2017).

\subsection{Coleta de Dados}

A coleta de dados foi realizada na Mina de Alegria em Mariana, MG. As estruturas dos TC foram fotografadas com uma câmera comum RGB de dimensões $4000 \times 2000$ pixels. As imagens foram coletadas em ângulos diferentes sempre com foco na parte central das estruturas, local onde há mais acúmulo dos resíduos (Figura 3).

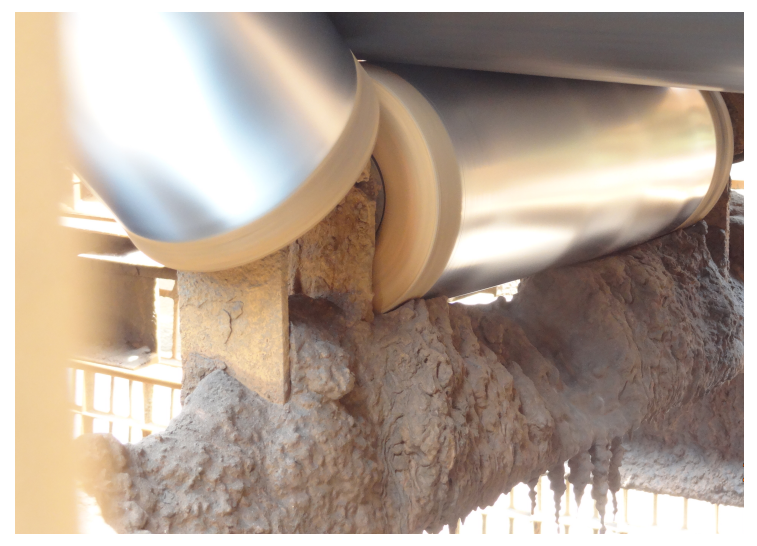

Figura 3. Estrutura do TC com acúmulo de resíduos.

Como a pesquisa está em fase inicial, foram definidas duas classes principais para análise do problema: (i) Limpo e (ii) Sujo. Essas classes caracterizam um problema binário e servirão de base para uma primeira avaliação de viabilidade do sistema proposto com RNC. Ao total, foram coletadas 73 imagens que formam o conjunto de dados inicial.

\subsection{Pré-processamento das Imagens}

Após a coleta dos dados, as imagens foram separadas conforme suas classes e, antes de serem inseridas na RNC para treinamento, elas foram pré-processadas com a seguinte metodologia:

(1) Resize: As imagens foram redimensionadas para um tamanho de $256 \times 256$ pixels;

(2) Random Crop: Um corte aleatório na imagem foi realizado com tamanho de $224 \times 224$ pixels.

Essas opções de pré-processamento foram escolhidas pois as RNC utilizadas tem entradas $224 \times 224$ pixels. Logo, a redução para $256 \times 256$ reduz a imagem sem perca de regiões importantes da mesma e o corte ajusta o tamanho do dado de entrada para a rede. 


\subsection{Data Augmentation}

O uso da técnica de Data Augmentation (DA) produz algumas alterações nas imagens no processo de treinamento e possibilita o melhor uso das imagens quando se tem poucos dados (Bhatia et al., 2019). Como o conjunto original de dados possui apenas 73 imagens, algumas técnicas de DA foram utilizadas para aumentar apenas o conjunto de treinamento da rede:

(1) Random Horizontal Flip: Um giro horizontal foi aplicado nas imagens com uma probabilidade de ocorrência de 0.3 ;

(2) Random Vertical Flip: Um giro vertical foi aplicado nas imagens com uma probabilidade de ocorrência de 0.3

(3) Random Rotation: Uma inclinação aleatória com ângulo de até $30^{\circ}$ foi aplicada nas imagens;

(4) Color Jitter: Uma alteração aleatória de até 0.05 na saturação foi aplicada nas imagens;

Essas alterações são aplicadas de forma automática pelo pytorch, a cada época de treinamento, em cada batch de dados. Dessa forma não há como calcular de forma exata quantas imagens diferentes tem o conjunto de treinamento ao fim do processo. A Figura 4 apresenta o préprocessamento e DA aplicado em um conjunto de imagens. Pode-se verificar nas imagens as alterações citadas anteriormente.

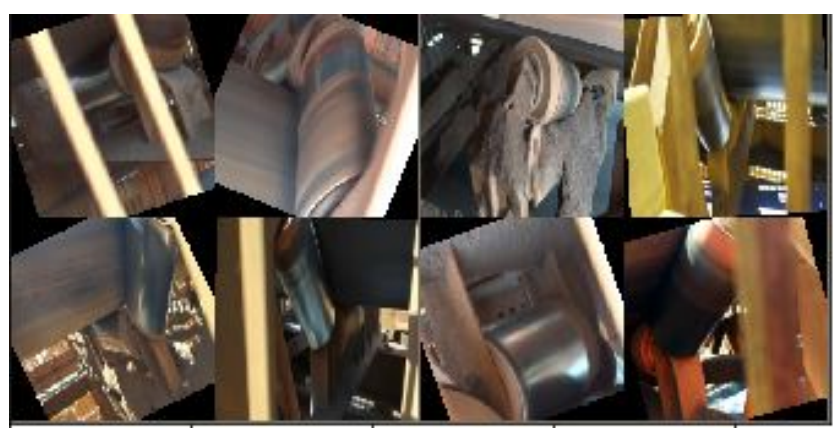

Figura 4. Pré processamento e DA aplicados nas imagens.

\subsection{Rede Neural Convolucional}

Para o treinamento e classificação de problemas por imagem, a estrutura de uma RNC possibilita a extração de características importantes do conjunto de dados.

O uso da técnica de Transfer Learning, ou Transferência de Conhecimento, viabiliza a construção de modelos precisos de redes de uma maneira que economiza tempo, (Bhatia et al., 2019). Assim esta técnica foi escolhida como meio para desenvolvimento da pesquisa.

Foram selecionadas duas redes para realizar a transferência de conhecimento, com base nas suas características de generalização: ResNet18 e VGG16. Segundo Simonyan and Zisserman (2014) e He et al. (2016), elas foram treinadas originalmente com o conjunto de dados ImageNet e apresentam ótimas características de generalização para vários conjuntos de dados.

O processo de treinamento foi realizado isonomicamente para ambas as redes através do método Finetuning (ajuste fino), no qual a rede é inicializada com os pesos originais, que são atualizados de acordo o treinamento. Foi alterado apenas a camada final de cada rede visando a adequação do problema com a duas classes a serem identificadas.

O treinamento das redes foi fixado em 100 épocas, e o modelo que apresentou melhor acurácia foi selecionado como melhor opção de rede. Os principais parâmetros de treinamento utilizados nas duas redes são apresentados a seguir.

- Batch Size: 8

- Optmizer - Stochastic Gradient Descent (SGD)

- Learning Rate: 0.001

- Decay: 0.1

- Step Size: 50

- Error - Cross Entropy Loss

Os dados coletados foram divididos aleatoriamente da seguinte forma: $70 \%$ para dados de treinamento e $30 \%$ para dados de teste. Assim, a quantidade de imagens para cada conjunto foi:

\section{- Imagens para treinamento: 51}

- Imagens para teste: 22

Com o objetivo de não deixar viés para algum tipo de classe, os dados de treinamento foram divididos da seguinte forma: 26 imagens para a classe Limpo e 25 imagens para a classe Sujo. Já os dados de teste foram divididos com: 12 imagens para a classe Limpo e 10 imagens para a classe Sujo.

\section{RESULTADOS}

As duas redes apresentaram resultados similares ao final dos treinamentos. São eles:

\subsection{Resultado ResNet18}

O treinamento com transferência de conhecimento do modelo ResNet18 foi realizado em 12 minutos e 04 segundos. O melhor índice de exatidão do conjunto de teste foi obtido com $81.81 \%$ de acerto, conforme Figura 5.

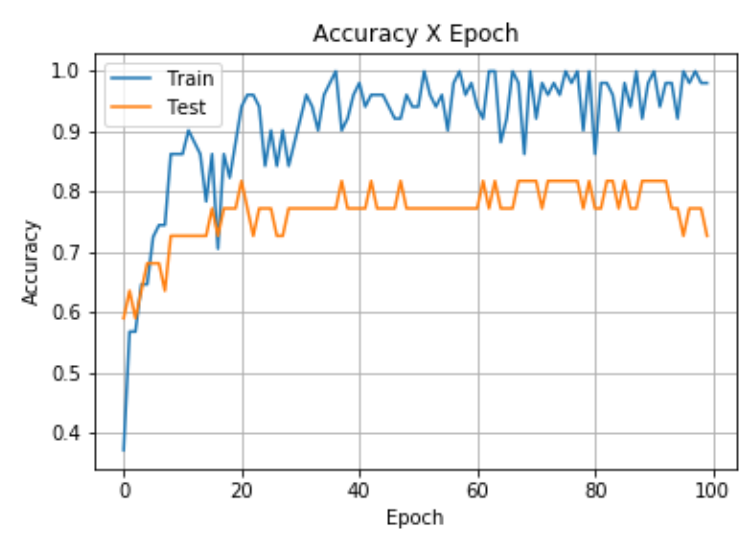

Figura 5. Índice de exatidão da rede ResNET18.

Com os dados de teste da rede, a matriz de confusão foi construída conforme Figura 6. Foi observado que 10 imagens da classe Limpo ( $45.45 \%$ do conjunto teste) foram classificadas corretamente, assim como 8 imagens da classe 
Sujo (36.36\% do conjunto teste). Quatro imagens, ao todo, foram classificadas com erro.

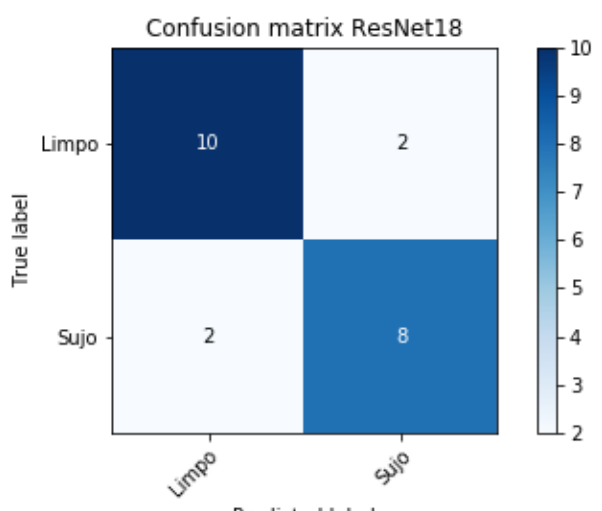

Predicted label

Figura 6. Matriz de confusão da rede ResNET18.

Uma comparação pode ser vista na Figura 7. Na primeira imagem há uma predição correta de um rolo com muito material aglutinante. Já na imagem ao lado, há a predição errada de uma estrutura limpa, prevista como suja. Observa-se neste caso em específico que a imagem não tem um foco específico no rolo do TC. Sugere-se que isso possa ter influenciado na predição errada da rede.
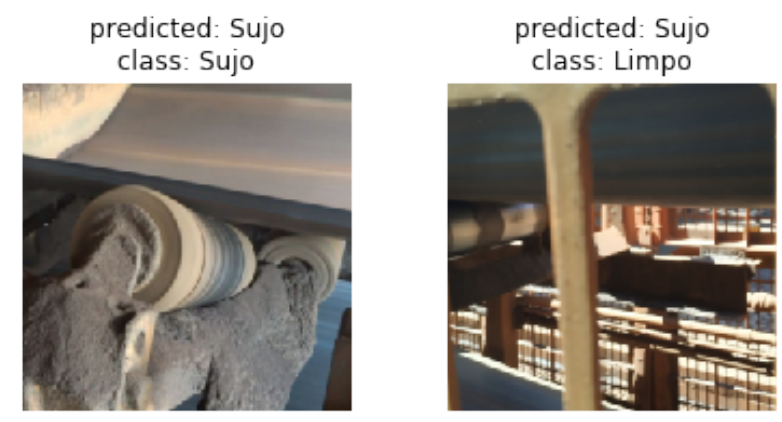

Figura 7. Predições da rede ResNET18.

\subsection{Resultado VGG16}

O treinamento com transferência de conhecimento da rede VGG16 foi realizado em 13 minutos e 54 segundos. O melhor índice de exatidão com os dados de teste foi obtido com $95.45 \%$ de acerto, na época 43, conforme Figura 8.

Com os dados de teste da rede, a matriz de confusão foi construída conforme Figura 9. Foi observado que 11 imagens da classe Limpo ( $50 \%$ do conjunto teste) foram classificadas corretamente, assim como 10 imagens da classe Sujo ( $45.45 \%$ do conjunto teste). Apenas uma imagem, ao todo, foi classificada com erro.

Na Figura 10 observa-se duas classificações corretas. As imagens estão focadas na região central do rolo do TC, sendo possível observar claramente a diferença entre os estados Limpo e Sujo. Com o modelo de rede VGG16, a única classificação errada se deu em função da imagem com maior distorção de características entre todas as 51 imagens de treinamento, que foi inserida propositalmente no conjunto teste.

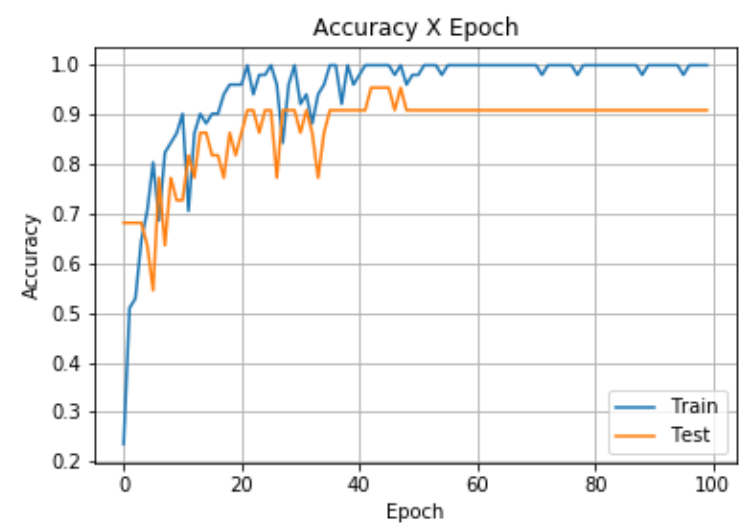

Figura 8. Índice de exatidão da rede VGG16.

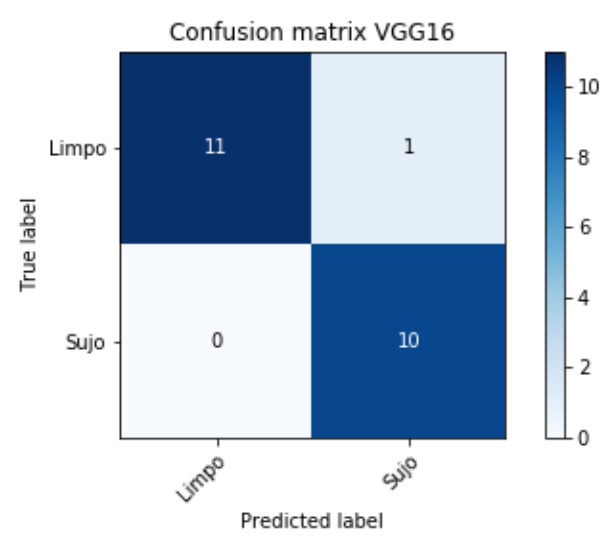

Figura 9. Matriz de confusão da rede VGG16.
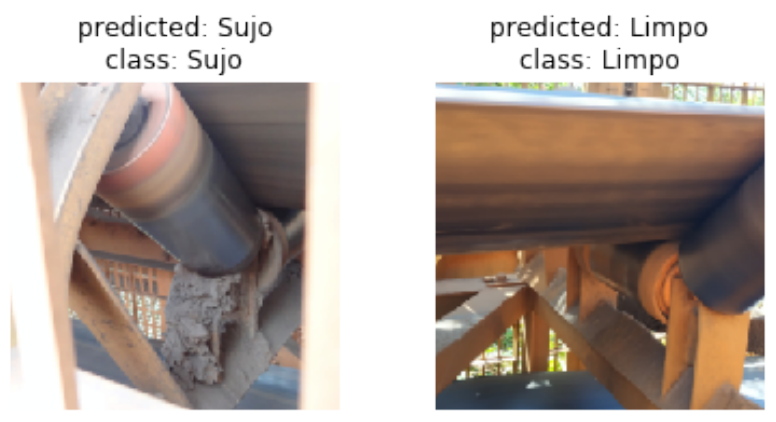

Figura 10. Predições da rede VGG16.

\subsection{Comparação dos Resultados}

Não foram encontrados na literatura outros trabalhos com o mesmo objetivo de classificação apresentado aqui: análise de materiais aglutinantes em estruturas de TC. Sendo assim, a comparação dos resultados foi realizada apenas entre as próprias redes treinadas.

As redes apresentaram resultados iniciais com taxa de exatidão variando entre $81 \%$ e $95 \%$. Este resultado indica a viabilidade da continuação e aprimoramento da pesquisa.

Foi observado que o uso de transferência de conhecimento é importante para este tipo de situação, e que as redes apresentaram boa capacidade de reconhecer os dados após 
o treinamento. No entanto, vale salientar que a quantidade de dados de treinamento foi pequena, e que o aumento desses dados pode elevar a capacidade de generalização da rede.

Estabelecendo uma comparação direta entre as redes ResNet18 e VGG16, a rede VGG16 se mostrou mais eficiente do que a ResNet18 para o conjunto testado. No entanto, análises com mais dados de treinamentos e alterações nos parâmetros de treinamento e dos próprios modelos de redes podem fazer com que esses resultados sejam maximizados.

\section{CONCLUSÃO}

A detecção automática da aglomeração de resíduos usando IA pode auxiliar no processo de inspeção dos TC ao diminuir o tempo de operação, ajudando na tomada de decisão dos setores de manutenção.

Os primeiros resultados dessa análise preliminar de aglutinantes em TC, por meio de RNC, são positivos. Foi verificado que o uso da transferência de conhecimento é eficiente para este tipo de problema, viabilizando um treinamento em curto período de tempo.

As redes treinadas com base na ResNet18 e VGG16 alcançaram resultados de exatidão nos teste acima de $80 \%$, em ambos os casos. O melhor índice de desempenho foi da rede VGG16 com $95 \%$ de acerto. Desta forma, verifica-se que essas redes são eficientes para este tipo de classificação. Assim, é importante expor as vantagens que a IA, aplicada na inspeção de TC, pode proporcionar ao processo de manutenção. A não detecção dos resíduos em tempo hábil pode implicar numa parada não programada da planta.

Os resultados preliminares foram promissores e dão suporte para que novos ensaios sejam realizados após a construção de um conjunto maior e mais diversificado de dados. Com a continuidade da pesquisa, novos dados serão coletados em condições mais adversas. Além disto, a alteração da estrutura interna de algumas camadas das RNC será estudada com o objetivo de maximizar a generalização do modelo treinado e a efetividade da classificação.

\section{REFERÊNCIAS}

Aghdam, H.H. and Heravi, E.J. (2017). Guide to convolutional neural networks: a practical application to trafficsign detection and classification. Springer.

Bhatia, Y., Rai, R., Gupta, V., Aggarwal, N., Akula, A., et al. (2019). Convolutional neural networks based potholes detection using thermal imaging. Journal of King Saud University-Computer and Information Sciences.

Chen, H., Pang, Y., Hu, Q., and Liu, K. (2018). Solar cell surface defect inspection based on multispectral convolutional neural network. Journal of Intelligent Manufacturing, 1-16.

Eletrobrás, Procel, Procel Indústria, Instituto Euvaldo Lodi, and CNI (2009). Correias transportadoras: guia básico. IEL/NC.

Guastala, F.A. (2013). Estudo do efeito da degradação térmica nas propriedades mecânicas de correias transportadoras operando em alta temperatura. Programa de Pós-Graduação em Engenharia de Materiais. Rede Temática em Engenharia de Materiais.
Haykin, S.S., Haykin, S.S., Haykin, S.S., and Haykin, S.S. (2009). Neural networks and learning machines, volume 3. Pearson Upper Saddle River.

He, K., Zhang, X., Ren, S., and Sun, J. (2016). Deep residual learning for image recognition. In Proceedings of the IEEE conference on computer vision and pattern recognition, 770-778.

Janssens, O., Schulz, R., Slavkovikj, V., Stockman, K., Loccufier, M., Van de Walle, R., and Van Hoecke, S. (2015). Thermal image based fault diagnosis for rotating machinery. Infrared Physics \& Technology, 73, 78-87.

Loures, M.M. (2010). Sistema automático de detecção de falhas em rolamentos de roletes de correias transportadoras. Universidade Federal de Minas Gerais.

Luque-Vega, L.F., Castillo-Toledo, B., Loukianov, A., and Gonzalez-Jimenez, L.E. (2014). Power line inspection via an unmanned aerial system based on the quadrotor helicopter. In MELECON 2014-2014 17th IEEE Mediterranean Electrotechnical Conference, 393-397. IEEE.

Malagoni, R.A. and dos Santos, D.A. (2014). Projeto de correias transportadoras: um estudo computacional de comparação dos métodos CEMA e prático, volume 3.

Masci, J., Meier, U., Ciresan, D., Schmidhuber, J., and Fricout, G. (2012). Steel defect classification with maxpooling convolutional neural networks. In The 2012 International Joint Conference on Neural Networks (IJCNN), 1-6. IEEE.

Máthé, K. and Buşoniu, L. (2015). Vision and control for uavs: A survey of general methods and of inexpensive platforms for infrastructure inspection. Sensors, 15(7), 14887-14916.

Matthee, H. (2009). Using ir imaging to diagnose problems in conveyors and crushers. Engineering and Mining Journal, 210(2), 38.

Paszke, A., Gross, S., Chintala, S., Chanan, G., Yang, E., DeVito, Z., Lin, Z., Desmaison, A., Antiga, L., and Lerer, A. (2017). Automatic differentiation in pytorch. In NIPS- $W$.

Qiao, T., Zhao, B., Shen, R., and Zheng, B. (2013). Infrared image detection of belt longitudinal tear based on svm.

Riese, F.M. and Keller, S. (2019). Soil texture classification with 1d convolutional neural networks based on hyperspectral data. arXiv preprint arXiv:1901.04846.

Simonyan, K. and Zisserman, A. (2014). Very deep convolutional networks for large-scale image recognition. arXiv preprint arXiv:1409.1556.

Toffolo, T.A.M. (2009). Otimizaçao do fluxo de produtos de uma empresa mineradora.

Vale (2019a). Carajás. URL http://www.vale.com/ brasil/PT/business/mining/iron-ore-pellets/ Documents/carajas/index.html.

Vale (2019b). Minério de ferro e pelotas. URL http://www.vale.com/brasil/PT/business/mining/ iron-ore-pellets/Paginas/default.aspx.

Zou, H. and Huang, F. (2015). A novel intelligent fault diagnosis method for electrical equipment using infrared thermography. Infrared Physics \& Technology, 73, 2935. 